\title{
Pruebas diagnósticas para la COVID-19: la importancia del antes y el después
}

Priscilia Aguilar Ramírez* 1; Yanina Enriquez Valencia 1; Carlos Quiroz Carrillo 1; Edward Valencia Ayala 1; Joel de León Delgado ${ }^{1}$; Arturo Pareja Cruz ${ }^{1}$

\section{RESUMEN}

En 2019, en la cuidad de Wuhan, China, se detectaron pacientes infectados con un nuevo coronavirus denominado 2019-nCoV, hoy SARS-CoV-2. El alarmante crecimiento de esta pandemia hace necesario conocer e implementar métodos de diagnóstico confiables para detectar y tratar adecuadamente a los pacientes, lo que contribuirá a frenar la propagación de la enfermedad. La necesidad de tener profesionales entrenados en la toma de muestras, en las buenas prácticas de laboratorio clínico y en el manejo de técnicas moleculares y hematológicas es de suma importancia para identificar adecuadamente la infección con este virus. La estandarización de los protocolos de obtención, traslado y almacenamiento de la muestra es igualmente relevante para detener la expansión de la pandemia de COVID-19 causada por el SARS-CoV-2.

Palabras clave: Infecciones por coronavirus; Diagnóstico; RT-PCR (Fuente: DeCS BIREME).

\section{Diagnostic tests for COVID-19: the importance of the before and the after}

\section{ABSTRACT}

In 2019, in the city of Wuhan, China, patients were diagnosed with a novel coronavirus originally named 2019-nCoV, currently known as SARS-CoV-2. The alarming expansion of this pandemic makes it necessary to find out and implement reliable diagnostic methods in order to properly detect and treat patients, thus contributing to slowing down the spread of the disease. It is extremely important to have trained professionals in sample collection, good clinical laboratory practices, and molecular and hematological techniques to adequately detect any cases of infection with this virus. Moreover, standardized protocols are essential for obtaining, transferring and storing samples to stop the spread of the COVID-19 pandemic caused by SARS-CoV-2.

Keywords: Coronavirus infections; Diagnosis; RT-PCR (Source: MeSH NLM).

1 Universidad de San Martín de Porres, Facultad de Medicina Humana, Instituto de Investigación, Centro de Investigación de Infectología e Inmunología. Lima, Perú.

* Autor corresponsal 


\section{INTRODUCCIÓN}

En diciembre del 2019, en la ciudad de Wuhan, provincia de Hubei en China, se originó un brote de casos de neumonía de origen desconocido, enfermedad denominada, actualmente, COVID-19 ${ }^{(1)}$. El aumento progresivo de casos, que supera los dos millones de infectados en casi todos los países, llevó a la OMS hace algunas semanas a declarar esta infección viral como pandemia. La determinación de la secuencia genómica del virus que la ocasiona (Gen Bank, código de acceso MN908947.3) (2) permitió identificar la relación entre este y los miembros de una especie viral denominada CoV causantes del síndrome respiratorio agudo severo (SARS, por sus siglas en inglés) ${ }^{(3-5)}$, por lo que el virus fue denominado SARS-CoV-2 por el Comité Internacional de Taxonomía de Virus ${ }^{(6)}$. En la gran mayoría de los casos, la infección por SARS-CoV-2 presenta síntomas parecidos a la influenza, pero en los pacientes con edad avanzada la enfermedad puede progresar hasta una forma grave de neumonía intersticial, que rápidamente evoluciona y causa la muerte a entre el $2 \%$ y el $5 \%$ de los casos, aunque factores como la edad y las enfermedades crónicas preexistentes pueden incrementar esta cifra ${ }^{(1)}$.

Debido a su condición de pandemia, es imprescindible contar con métodos de diagnóstico confiables para la determinación de esta infección viral, lo que contribuye a su diagnóstico oportuno, y además reduce la posibilidad de clasificar a individuos como falsos negativos, los que podrían propagar la enfermedad. Diversos institutos de investigación en todo el mundo, asociados con laboratorios de salud pública, son capaces de implementar tecnologías de detección basadas en la reacción en cadena de la polimerasa de transcripción inversa en tiempo real (RT-PCR, por sus siglas en inglés), y pruebas serológicas basadas en la detección de las inmunoglobulinas específicas de cepas de coronavirus para emitir diagnósticos precisos y confiables. En todos estos casos, las muestras virales de pacientes infectados son la única fuente que se tiene para establecer, controlar ensayos y validar protocolos que son compartidos con la comunidad internacional.

Si bien los exámenes de laboratorio son determinantes para el diagnóstico de la enfermedad, no menos relevante es realizar una buena toma de muestra al paciente, procedimiento que contribuirá a la conservación de la muestra y la fiabilidad del resultado final. En este sentido, los errores de diagnóstico como consecuencia de incorrectos procedimientos preanalíticos pueden ocurrir en los laboratorios clínicos, sobre todo cuando el personal está sometido a entregar resultados bajo alta presión laboral, tal como ocurre en los laboratorios en todo el mundo debido al crecimiento exponencial de casos positivos a SARS-CoV-2 ${ }^{(7)}$.

El presente trabajo muestra una breve introducción sobre la estructura, la filogenética y la respuesta inmune del hospedero contra los coronavirus, información necesaria para diseñar y validar diversas técnicas de diagnóstico para la COVID-19. Adicionalmente, hace referencia a temas relacionados con la toma y manejo de la muestra, aspectos no menos relevantes de la etapa preanalítica del diagnóstico.

\section{FAMILIA DE LOS CORONAVIRUS}

\section{Estructura de los coronavirus}

Los coronavirus son una familia de virus que constan de una envoltura proteica esférica, con un tamaño que varía entre 80 y $120 \mathrm{~nm}$. Su información genética está codificada en una cadena simple de ARN (ssRNA, por sus siglas en inglés) que mide, según el género, de 26,2 a 31,7 kb. Su genoma está compuesto por seis a diez marcos de lectura abiertos (ORF, por sus siglas en inglés). El primer ORF ocupa los dos tercios de todo el genoma viral y codifica la proteína ARN dependiente de ARN polimerasa (RdRp), mientras que el último tercio solo tiene genes de proteínas estructurales. La cubierta viral incluye tres proteínas: la proteína de envoltura $(E)$, la proteína de membrana $(M)$ y la proteína de espiga (S). Las dos primeras se encargan del ensamblaje viral, mientras que la $S$, que presenta un dominio de unión al receptor celular, es la encargada de liberar el genoma viral dentro de la célula que va a ser infectada. Adicionalmente, presenta una proteína que está asociada al ARN viral (proteína del nucleocápside-N) y muchos miembros de esta familia viral también expresan la proteína hemaglutinina esterasa $(\mathrm{HE})^{(8,9)}$.

El ingreso del virus depende de la interacción entre el vibrión y la célula huésped. La infección es iniciada por la unión de la proteína $\mathrm{S}$ con diversas proteínas específicas en la superficie celular (Neu 5,9 Ac2, CEACAM1, entre otras) aunque la enzima convertidora de angiotensina (ACE-2, por sus siglas en inglés) resulta ser especialmente relevante. Después, los virus envueltos fusionan su envoltura con la membrana de la célula huésped para liberar su nucleocápside, lo que muestra el doble papel de la proteína S: mediar en la unión y la fusión de membranas (10-12).

\section{Filogenética de los coronavirus}

En la familia de coronavirus se incluyen los géneros Alfacoronavirus, Betacoronavirus, Gammacoronavirus y Deltacoronavirus. Loscuatrosonconsiderados comoreservorios naturales y vectores de una variedad de coronavirus. Estos virus han cruzado las barreras de las especies para infectar a muchos otros tipos diferentes de animales, incluidos aviares y roedores. De estos géneros, el que tiene mayor capacidad de infectar humanos es el Betacoronavirus conformado por coronavirus detectados en mamíferos como bovinos, equinos, porcinos, caninos, murinos, murciélagos y humanos ${ }^{(13)}$. 
En este año, el género fue ampliado debido al continuo brote de neumonía en la ciudad de Wuhan, donde se estudiaron varias familias con diversos síntomas asociados a la enfermedad, como fiebre, malestar en los tractos respiratorios superior e inferior, diarrea, linfopenia, trombocitopenia y aumento de los niveles de proteína $C$ reactiva y de lactato deshidrogenasa, sin presentar ninguna infección bacteriana. Debido a la falta de una respuesta, se realizaron exámenes radiológicos que mostraron opacidades pulmonares descritas como "vidrio esmerilado". Al realizar el ensayo de los 5 genes proteicos expresados por los coronavirus se decidió amplificar, por RT-PCR, los genes que codifican las proteínas RdRp y $\mathrm{S}$, y luego efectuar su secuenciamiento. Se identificó así un nuevo tipo de coronavirus, muy cercano al Rhinolophus murciélago virus, al que denominaron inicialmente 2019-nCoV, hoy conocido como SARS-CoV-2 ${ }^{(14)}$.

Respecto a su secuencia genética, el SARS-CoV-2 comparte una homología del $88 \%$ con dos coronavirus aislados de murciélagos que pertenecen a este mismo género. También se identificó una homología del $79 \%$ con el virus SARS-CoV y un $50 \%$ con el virus Human MERS-CoV. Esta diferencia con el SARS-CoV sirvió para incluir a esta especie como un nuevo miembro del género Betacoronavirus. El secuenciamiento genómico del gen de la proteína $S$ del nuevo coronavirus muestra que contiene más nucleótidos que el gen de las especies Rousettus murciélago coronavirus HKU4, que el SARS-CoV y el MERS-CoV. Sin embargo, esta proteína también utiliza a la ACE-2 como uno de los receptores que media su ingreso a la célula ${ }^{(12-14)}$.

\section{ENSAYOS MOLECULARES EN EL DIAGNÓSTICO DE COVID-19}

El examen molecular que se está empleando en todo el mundo para la detección directa de la infección con el SARS-CoV-2 es la prueba de RT-PCR sobre los genes expresados por este virus. Liu et al. analizaron, por medio de RT-PCR, la expresión génica de los fragmentos del SARS-CoV-2 a partir de muestras de esputo, lavado broncoalveolar e hisopado del tracto respiratorio provenientes de 4880 pacientes con síntomas de infección respiratoria o contacto cercano con el virus en el hospital de Wuhan. Se realizó el secuenciamiento del fragmento génico ORF1ab y de un fragmento de la proteína de la nucleocápside (NP). Para un diagnostico confirmatorio, los autores consideraron como pacientes SARS-CoV positivos a los que presentaron ambos fragmentos génicos. Para el esputo, el fragmento NP fue expresado en el 49,12\% de pacientes mientras que el fragmento ORF1ab en el 50,88 \%, y el porcentaje de pacientes que expresaron ambos fragmentos fue del $49,12 \%$. En el lavado broncoalveolar, el fragmento NP fue expresado en el $80 \%$ de pacientes, mientras que el fragmento ORF 1 ab en el $100 \%$, y el porcentaje de pacientes que expresaron ambos fragmentos fue del $80 \%$. En el caso del hisopado del tracto respiratorio, el fragmento NP fue expresado en el $39,8 \%$ de pacientes, mientras que el fragmento ORF1ab en el 40,98\%, y el 38,42\% de los pacientes expresaron ambos fragmentos. De estos resultados, encontraron que los pacientes más expuestos son los adultos mayores de 70 años con el $61,81 \%$ de incidencia. Por otro lado, un grupo de pacientes tuvieron alguna sintomatología asociada al coronavirus, pero fueron descartados ya que su resultado salió negativo, lo que mostró la eficacia de esta técnica en la identificación del SARS-CoV-2 ${ }^{(15)}$.

Corman et al. propusieron una prueba diagnóstica que discrimina 2019-nCoV de SARS-CoV, mediante el secuenciamiento de 297 muestras clínicas que contienen todo el espectro de virus respiratorios humanos. Para esto se diseñaron cebadores para el secuenciamiento génico de los siguientes fragmentos: 1) ORF1ab-gen RdRp en la posición 15361-15460 nts; 2) gen de la proteína E, posición 26141-26253 nts, y 3) gen de la proteína N posición 28555-28682 nts. Los cebadores fueron diseñados en base al secuenciamiento de la primera muestra obtenida en la provincia de Wuhan en diciembre de 2019 (NM908947 Wuhan-Hu-1) y de otras muestras virales (NC-004718 SARS-CoV). Se demostró que estos cebadores diseñados se acoplan a varias especies SARS-CoV, lo que indica que todas las muestras SARS son Betacoronavirus. Por otro lado, decidieron alinear las secuencias génicas de todas las muestras analizadas: 1) las muestras de la ciudad de Wuhan nombrada como BetaCoV/Wuhan/PBCAMSWH-01/2019/EPI_ISL_402123; 2) Bat SARS CoV (murciélago) nombrado para el ensayo como bat-SL-CoVZC45 (número de acceso GenBank MG772933), así como 3) el miembro más distante dentro del CoV de murciélago relacionado con el SARS detectado en Bulgaria, el Bat coronavirus BM48-31/ BGR/2008 (número de acceso de GenBank NC_014470). No se encontraron diferencias en la secuencia nucleotídica para la proteína $\mathrm{E}$, pero sí en los genes RdRp y N, lo que muestra que existen diferencias en la secuencia nucleotídica en los virus SARS, lo que sirve como una prueba de diferenciación de una especie viral frente a otra ${ }^{(16)}$.

Hace algunos años se diseñaron cebadores de los fragmentos localizados en ORF1ab (gen de la proteína 1A posición 11197-11280 nts, gen RdRpSeq posición 15049-15290 nts, ORF1b posición 18266-18347 nts), del gen de la proteína $\mathrm{E}$ (upE posición $27458-27550 \mathrm{nts}$ ) y, finalmente, del fragmento NSeq posición 29549 a29860 nts para determinar diferencias entre las distintas cepas de coronavirus humano (hCoVEMC). El secuenciamiento mostró homología en todos los genes, excepto en las secuencias RdRpSeq y Nseq en las que se observan diferentes polimorfismos (inserción/deleción) a lo largo del gen, lo que indica que solo el análisis de estos genes (RdRpSeq y Nseq) es suficiente para diferenciar diversas cepas de coronavirus. En consecuencia, es necesario estandarizar protocolos en el diseño de cebadores que detecten diferencias entre las secuencias con el fin de diferenciar un coronavirus de otro ${ }^{(17)}$. 


\section{Protocolos estandarizados y precisión en el diagnóstico molecular}

Según la OMS, en el caso del SARS-CoV-2, la detección del gen de la proteína $\mathrm{E}$ se emplea como primera prueba confirmatoria, seguida de la expresión del gen RdRp. La expresión del gen $\mathrm{N}$ solo se usa si se requiriese un ensayo confirmatorio adicional. Para los Centros para el Control y la Prevención de Enfermedades (CDC, por sus siglas en inglés), la primera prueba confirmatoria sería la expresión y secuenciamiento del gen $\mathrm{N}$ diseñado para la detección universal de los coronavirus SARS y del SARS-CoV-2, para lo cual se utilizan dos cebadores diferentes ${ }^{(18)}$ (Tabla 1).

Tabla 1. Comparación del ensayo de diagnóstico molecular por RT-PCR realizado por la OMS y por los CDC para diagnosticar el SARS-CoV-2

\begin{tabular}{|c|c|c|c|c|c|}
\hline Ensayo & Gen & Alcance & $\begin{array}{l}\text { Límite inferior } \\
\text { detectable }\end{array}$ & Muestras & $\begin{array}{l}\text { Condiciones de } \\
\text { almacenamiento }\end{array}$ \\
\hline \multirow[t]{3}{*}{ OMS } & $E$ & $\begin{array}{l}\text { Primer } \\
\text { ensayo }\end{array}$ & $\begin{array}{l}3,9 \text { copias } x \\
\text { reacción }\end{array}$ & \multirow{3}{*}{$\begin{array}{l}\text { Hisopo nasofaríngeo y } \\
\text { orofaríngeo o muestras } \\
\text { de tracto respiratorio } \\
\text { inferior (esputo, aspirado } \\
\text { endotraqueal y/o lavado } \\
\text { broncoalveolar) }\end{array}$} & $\leq 5$ días $\left(2\right.$ a $\left.8^{\circ} \mathrm{C}\right)$ \\
\hline & RdRp & $\begin{array}{l}\text { 1. er ensayo } \\
\text { confirmatorio }\end{array}$ & $\begin{array}{l}3,6 \text { copias } x \\
\text { reacción }\end{array}$ & & $>5$ días $\left(\leq 70^{\circ} \mathrm{C}\right.$; hielo seco) \\
\hline & N & $\begin{array}{l}\text { 2. do ensayo } \\
\text { confirmatorio }\end{array}$ & $\mathrm{N} / \mathrm{A}$ & & \\
\hline \multirow{3}{*}{$\mathrm{CDC}$} & $\mathrm{N} 1 / 2 / 3$ & Ensayo & $1,0-3,2$ copias $/ \mu \mathrm{L}$ & \multirow{3}{*}{$\begin{array}{l}\text { Hisopos nasofaríngeos y } \\
\text { orofaríngeos, esputo, } \\
\text { aspirados del tracto } \\
\text { respiratorio inferior, } \\
\text { lavado broncoalveolar } \\
\text { y lavado nasofaríngeo o } \\
\text { aspirado nasal }\end{array}$} & $\leq 4$ días $\left(4^{\circ} \mathrm{C}\right)$ \\
\hline & & combinado & & & $>4$ días $\left(\leq 70^{\circ} \mathrm{C}\right)$ \\
\hline & RNase $\mathrm{P}$ & Ensayo control & $\mathrm{N} / \mathrm{A}$ & & \\
\hline
\end{tabular}

E: gen de envoltura

N: gen del nucleocápside

RdRp: RNA dependiente de la RNA polimerasa

RNase P: gen humano RNase P

Traducido de Lipi et al., 2020

Los diagnósticos muchas veces no son certeros y no es a causa del protocolo de ensayo o por el personal técnico, sino porque dependen de la carga viral y de si el paciente ha recibido o no tratamiento. Ai et al. realizaron repetidas pruebas en tomografías computarizadas seguidas de RT-PCR a 1014 pacientes con COVID-19 en Wuhan, China. En estas pruebas se analizó la conversión dinámica de los resultados de RT-PCR (negativo a positivo, positivo a negativo, respectivamente) así como las tomografías computarizadas (TC) del tórax de cada paciente. En un primer momento, el $59 \%$ tuvo resultados positivos de RT-PCR y $88 \%$ tuvo TC de tórax positiva, este último con una sensibilidad del $97 \%$. De los 415 pacientes negativos de RT-PCR, el 75 \% fueron positivos en la TC de tórax y considerados como pacientes que iban a desarrollar la enfermedad. Por otro lado, mediante los ensayos seriales de RT-PCR y tomografías computarizadas, se observó que el tiempo de intervalo medio entre los resultados iniciales negativos de RT-PCR fue de 5,1 $\pm 1,5$ días; mientras que el resultado inicial positivo a posterior negativo de RT-PCR fue de $6,9 \pm 2,3$ días. Además, si los pacientes mostraron mejoría en las tomografías computarizadas de tórax, los resultados de RT-PCR se volverían negativos. Lo anterior reafirma que los ensayos de RT-PCR deben ser acompañados de una tomografía computarizada, ya que durante la hospitalización resultados negativos se convirtieron gradualmente en positivos, y estos en negativos según el tratamiento de los pacientes ${ }^{(19)}$. Ya que RT-PCR puede producir resultados falsos negativos en la etapa inicial de la infección, se sugiere que los pacientes negativos a RT-PCR, con evidencias imagenológicas de alteraciones pulmonares, deben ser aislados y, posteriormente, repetir el ensayo molecular ${ }^{(20)}$.

La sensibilidad analítica de la RT-PCR se ve influida por la baja carga viral en pacientes asintomáticos o levemente sintomáticos, lo que ocurre, fundamentalmente, en dos momentos: 1) la fase inicial de la infección, cuando el paciente todavía es completamente asintomático o solo levemente sintomático, y 2) cuando la infección por SARS-CoV-2 es controlada por el sistema inmune, y los 
síntomas se alivian, con la consecuente eliminación de virus aún persistentes. Otro problema es la aparición de mutaciones que son atribuibles a las ARN polimerasas dependientes de ARN propensas a errores de coronavirus. Shen et al. realizaron el secuenciamiento del transcriptoma del lavado broncoalveolar de pacientes con SARS-CoV-2. Este análisis mostró variantes intrahospitalarias en los pacientes infectados con SARS-CoV-2. La distribución de las variantes génicas es similar en las poblaciones infectadas. Sin embargo, se observaron cuatro variantes virales intraindividuales en forma de polimorfismo ${ }^{(21)}$.

Hasta el momento, no se ha confirmado si la trasmisión de persona a persona induce a la variación en las secuencias genéticas del SARS-CoV-2, pero tampoco hay evidencias que la nieguen. Si ocurriera, la evolución in vivo después de la infección puede afectar su virulencia, infectividad y transmisibilidad, por lo que es necesario fortalecer la vigilancia. Si a estos problemas se le agregan los causados por las inadecuadas prácticas durante la obtención y manipulación de la muestra, el no escoger adecuadamente la ventana viral (por ejemplo, obtener la muestra cuando el paciente está en tratamiento), utilizar cebadores mal sintetizados, o no saber interpretar los perfiles de expresión génica, se corren grandes riesgos para obtener un diagnóstico certero ${ }^{(19)}$.

\section{ENSAYOS SEROLÓGICOS EN EL DIAGNÓSTICO DE LA COVID-19}

En la respuesta inmune antiviral contra los virus que afectan el tracto respiratorio, incluido el SARS-CoV-2, se involucran componentes moleculares y celulares del sistema innato y adquirido. Estos incluyen mediadores moleculares como los interferones tipo I y los anticuerpos IgA, IgM e IgG, así como neutrófilos, macrófagos y linfocitos con actividad citotóxica sobre las células infectadas, como los asesinos naturales y los T CD8+. Adicionalmente, las células dendríticas y los linfocitos auxiliadores T CD4+ son muy relevantes en la activación de la respuesta inmune efectiva. Específicamente, la secreción de anticuerpos es un indicador muy relevante y simple de detectar que refleja la respuesta inmune frente a los antígenos virales ${ }^{(22,23)}$.

Las pruebas rápidas serológicas son exámenes inmunocromatográficos o de inmunoensayo de flujo lateral, sencillos y muy fáciles de realizar que detectan, en un solo paso, los anticuerpos contra el virus. Para estas pruebas se puede utilizar muestras de suero, plasma o sangre total. Estas pruebas rápidas son complementarias y no sustituyen la detección del material genético viral por RT-PCR. En este sentido, una de las limitaciones del diagnóstico basado en la detección de inmunoglobulinas específicas a un determinado antígeno es la dificultad de conocer con certeza cuándo estas inmunoglobulinas aparecen en las muestras sanguíneas, lo que podría conducir a formular un diagnóstico falso negativo. Incluso, Dong et al. plantean que es tan compleja la sintomatología del perfil de COVID-19, que las pruebas serológicas de $\lg G$ e $\lg M$ especificas a SARS-CoV-2 no deben utilizarse como diagnóstico definitivo, sino que siempre es necesario el análisis molecular genético ${ }^{(24)}$.

Recientemente, Zhao et al. demostraron que en pacientes infectados con SARS-CoV-2 la presencia de anticuerpos IgM e IgG fue menor al $40 \%$ dentro de la primera semana desde el inicio de la infección y aumentó rápidamente hasta el $100 \%$ para el día 15 (presencia de IgM e IgG en el $94,3 \%$ y el $79,8 \%$ de los casos, respectivamente). En contraste, la detección del ARN disminuyó de 66,7 \% en las muestras recolectadas antes del día 7 al $45,5 \%$ durante los días 15-39. Es interesante observar que la combinación de detecciones de ARN y anticuerpos mejoró significativamente la sensibilidad del diagnóstico de la enfermedad COVID-19, incluso en la fase temprana de la infección. Este estudio evidenció que la respuesta aguda de anticuerpos en pacientes infectados con SARS-CoV-2 es muy similar a otras infecciones virales agudas. Demostró, además, que las pruebas serológicas pueden contribuir a lograr un diagnóstico oportuno, y que la detección de anticuerpos totales es mucho más sensible que IgM e IgG para detectar la infección ${ }^{(25)}$.

\section{BUENAS PRÁCTICAS EN LA OBTENCIÓN Y MANEJO DE LAS MUESTRAS PARA EL DIAGNÓSTICO DE LA COVID-19}

La bioseguridad es un aspecto muy importante que se debe considerar durante la toma y el manejo de la muestra requerida para el diagnóstico de la enfermedad COVID-19. La OMS recomienda el nivel 2 para realizar las pruebas de diagnóstico. El personal de laboratorio debe tener entrenamiento específico en el manejo de agentes patógenos, bajo la supervisión directa de un investigador competente, según las normas de bioseguridad de cada laboratorio.

Se ha considerado que las muestras del tracto respiratorio superior aumentan la sensibilidad de las pruebas moleculares, además de ser más fáciles de obtener. Para la toma de muestra, la OMS recomienda que el material se colecte con un hisopo de punta sintética (por ejemplo, nailon o dacrón) y un eje de aluminio o plástico. El procedimiento recomendado para recoger una muestra nasofaríngea de calidad implica insertar el hisopo y frotar en la fosa nasal paralela al paladar, manteniendo el hisopo en su lugar durante unos segundos para permitir la secreción y la absorción. Inmediatamente después se coloca el hisopo en un tubo estéril que contiene $2-3 \mathrm{ml}$ de medio de transporte viral. El procedimiento para recolectar muestras de la orofaringe (por ejemplo, garganta) implica frotar la faringe posterior, evitando la lengua e, inmediatamente, colocar el hisopo en otro tubo 
estéril separado que también contiene 2-3 $\mathrm{ml}$ de medios de transporte virales. Está demostrado que el uso incorrecto de los hisopos, la absorción inapropiada de material de diagnóstico y la inserción en viales inadecuados pueden causar errores de diagnóstico ${ }^{(26,27)}$.

Los errores preanalíticos en los estudios pueden ocurrir por falta de identificación o identificación errónea de la muestra, la colección inadecuada o la cantidad insuficiente de la muestra por analizar, las condiciones imprecisas de transporte y almacenamiento de la muestra (exposición a lesiones, cadena de frío poco confiable, tiempo de transporte prolongado) y la presencia de sustancias interferentes (por ejemplo, liberación de componentes celulares que pueden interferir en el ensayo debido a la congelación de sangre entera, uso de aditivos inapropiados) ${ }^{(28,29)}$. La viabilidad de la muestra es responsabilidad del personal que la recoge y que debe asegurar la cadena de custodia hasta que dicha muestra llega al laboratorio. Cuando el laboratorio que realiza el diagnóstico no es el responsable del proceso de toma de la muestra, deberá solicitar toda la información importante para el manejo, embalaje y condiciones de transporte de la muestra. Además, se debe contar con todos los datos relevantes que permitan la correcta identificación de la muestra (nombre y apellidos del usuario o código, fecha de nacimiento, tipo de muestra que se envía y fecha de recolección). Estos y otros detalles respecto al manejo de las muestras de pacientes se han protocolizado debido a su importancia en la condición pandémica actual (30).

\section{CONCLUSIONES}

Los protocolos de seguridad para la obtención, traslado, manejo y utilización de la muestra son de suma importancia para proporcionar resultados precisos e interpretables. Complementar las fortalezas de pruebas moleculares que permiten la detección específica del SARS-CoV-2 con ensayos inmunológicos que valoran la respuesta inmune del hospedero será crucial para el diagnóstico certero, y a tiempo, de los pacientes. Adicionalmente, estas mismas técnicas ofrecen alternativas para la determinación de marcadores moleculares con valor pronóstico, los que serán especialmente valiosos para diferenciar el manejo de los pacientes menores de 60 años sin comorbilidades preexistentes.

Contribución de los autores: Priscilia Aguilar, Yanina Enrique: redacción, revisión bibliográfica y edición. Carlos Quiróz y Edward Valencia: revisión bibliográfica. Joel de León: edición final. Arturo Pareja: edición, revisión final y coordinación general.

Fuentes de financiamiento: Este artículo ha sido financiado por los autores.

Conflicto de interés: Los autores declaran no tener ningún conflicto de interés.

\section{REFERENCIAS BIBLIOGRÁFICAS}

1. World Health Organization. Novel Coronavirus (2019-nCoV)Situation reports. 2020. Disponible en: https://www.who.int/ emergencies/diseases/novel-coronavirus-2019/situation-reports

2. GenBank. Severe acute respiratory syndrome coronavirus 2 isolate Wuhan-Hu-1, complete genome. GenBank. 2020.

3. Li C, Yang Y, Ren L. Genetic evolution analysis of 2019 novel coronavirus and coronavirus from other species. Infect Genet Evol. 2020; 82: 104285.

4. Ji W, Wang W, Zhao X, Zai J, Li X. Cross-species transmission of the newly identified coronavirus 2019-nCoV. J Med Virol. 2020; 92(4): 433-40.

5. Lu R, Zhao X, Li J, Niu P, Yang B, Wu H, et al. Genomic characterization and epidemiology of 2019 novel coronavirus: implications for virus origins and receptor binding. Lancet. 2020; 395(10224): 565-74.

6. Coronaviridae Study Group of the International Committee on Taxonomy of Viruses. The species severe acute respiratory syndrome-related coronavirus: classifying 2019-nCoV and naming it SARS-CoV-2. Nat Microbiol 2020; 5(2020): 536-44.

7. Lippi G, Plebani M, Graber ML. Building a bridge to safe diagnosis in health care: the role of the clinical laboratory. Clin Chem Lab Med. 2016; 54: 1-3.

8. Peiris JS, Yuen KY, Osterhaus AD, Stöhr K. The severe acute respiratory syndrome. N Engl J Med. 2003; 349(25): 2431-41.

9. Wu C, Liu Y, Yang Y, Zhang P, Zhong W, Wang Y, et al. Analysis of therapeutic targets for SARS- CoV-2 and discovery of potential drogs by computational methods. Acta Pharm Sin B. 2020.

10. Enjuanes L, Almazan F, Sola I, Zuniga S. Biochemical aspects of coronavirus replication and virus-host interaction. Annu Rev Microbiol. 2006; 60: 211-30.

11. Belouzard S, Millet JK, Licitra BN, Whittaker GR. Mechanisms of coronavirus cell entry mediated by the viral spike protein. Viruses. 2012; 4: 1011-33.

12. Walls AC, Park Y, Tortorici MA, Wall A, McGuire AT, Veesler D. Structure, function, and antigenicity of the SARS-CoV-2 spike glycoprotein. Cell. 2020.

13. Chan JF, Yuan S, Kok KH, To KK, Chu H, Yang J, et al. A familial cluster of pneumonia associated with the 2019 a study of a family cluster. Lancet. 2020; 395(10223): 514-23.

14. Zhou P, Yang XL, Wang XG, Hu B, Zhang L, Zhang W, et al. A pneumonia outbreak associated with a new coronavirus of probable bat origin. Nature. 2020; 579(7798): 270-3.

15. Liu R, Han H, Liu F, Lv Z, Wu K, Liu Y, et al. Positive rate of RT-PCR detection of SARS-CoV-2 infection in 4880 cases from one hospital in Wuhan, China, from Jan to Feb 2020. Clin Chim Acta. 2020; 505: 172-5.

16. Corman VM, Landt O, Kaiser M, Molenkamp R, Meijer A, Chu D, et al. Detection of 2019 novel coronavirus (2019-nCoV) by real-time RT-PCR. Euro Surveill. 2020; 25(3): 2000045.

17. Corman VM, Müller MA, Costabel U, Timm J, Binger T, Meyer B, et al. Assays for laboratory confirmation of Novel human Coronavirus (hCoV-EMC) Infections. Euro Surveill. 2012; 17(49).

18. Lippi G, Simundic AM, Plebani M. Potential preanalytical and analytical vulnerabilities in the laboratory diagnosis of coronavirus disease 2019 (COVID-19). Clin Chem Lab Med. 2020.

19. Ai T, Yang Z, Hou H, Zhan C, Chen C, Lv W, et al. Correlation of chest CT and RT-PCR testing in coronavirus disease 2019 (COVID19) in China: a report of 1014 cases. Radiology. 2020: 200642.

20. Long C, Xu H, Shen Q, Zhang X, Fan B, Wang C, et al. Diagnosis of the coronavirus disease (COVID-19): rRT-PCR or CT?. Eur J Radiol. 2020; 126: 108961. 
21. Shen Z, Xiao Y, Kang L, Ma W, Shi L, Zhang L, et al. Genomic diversity of SARS-CoV-2 in coronavirus disease 2019 patients. Clin Infect Dis. 2020.

22. Prompetchara E, Ketloy C, Palaga T. Immune responses in COVID-19 and potential vaccines: lessons learned from SARS and MERS epidemic. Asian Pac J Allergy Immunol. 2020; 38(1): 1-9.

23. Li G, Fan Y, Lai Y, Han T, Li Z, Zhou P, et.al. Coronavirus infections and immune responses. J Med Virol. 2020; 92(4): 424-32.

24. Dong $X$, Cao $Y$, Lu X, Zhang J, Du H, Yan Y, et al. Eleven faces of coronavirus disease 2019. Allergy. 2020.

25. Zhao J, Yuan Q, Wang H, Liu W, Liao X, Su Y, et al. Antibody responses to SARS-COV-2 in patients of novel coronavirus disease 2019. Clin Infect Dis. 2020.

26. Irving SA, Vandermause MF, Shay DK, Belongia EA. Comparison of nasal and nasopharyngeal swabs for influenza detection in adults. Clin Med Res. 2012; 10: 215-8.

27. Spencer S, Gaglani M, Naleway A, Reynolds S, Ball S, Bozeman S, et al. Consistency of influenza a virus detection test results across respiratory specimen collection methods using real-time reverse transcription-PCR. J Clin Microbiol. 2013; 51: 3880-2.

28. Espy MJ, Uhl JR, Sloan LM, Buckwalter SP, Jones MF, Vetter EA, et al. Real-time PCR in clinical microbiology: applications for routine laboratory testing. Clin Microbiol Rev. 2006; 19: 165-256.

29. Van Zyl G, Maritz J, Newman H, Preiser W. Lessons in diagnostic virology: expected and unexpected sources of error. Rev Med Virol. 2019; 29: e2052.

30. Centers for Disease Control and Prevention. Coronavirus Disease 2019 (COVID-19): Guidelines for Clinical Specimens. 2020. Disponible en: https:/ / www.cdc.gov/coronavirus/2019-ncov/lab/ guidelines-clinical-specimens.html

\section{Correspondencia:}

Priscilia Aguilar Ramírez

Teléfono: 3652300 (anexo 147)

Dirección: Av. Alameda del Corregidor 1531, Urb. Los Sirius, La Molina.

Lima, Perú. Correo electrónico: paguilarr1@usmp.pe

Recibido: 1 de abril de 2020.

Evaluado: 3 de abril de 2020.

Aprobado: 15 de abril de 2020.

(c) La revista. Publicado por Universidad de San Martín de Porres, Perú

(cc) $\mathbf{\text { Br }}$ Licencia de Creative Commons Artículo en acceso abierto bajo términos de Licencia Creative Commons Atribución 4.0 Internacional. (http://creativecommons.org/licenses/by/4.0/)

\section{ORCID iDs}

Joel De León Delgado O https: / / orcid.org/0000-0002-3664-8023

Arturo Pareja Cruz

Yanina Enriquez Valencia

(C) https: / / orcid.org/0000-0002-5988-5515

(C) https: / / orcid.org/0000-0002-8501-0915

Carlos Quiroz Carrillo

Edward Valencia Ayala
(D) https: / / orcid.org/0000-0002-4821-9496

(D https: / /orcid.org/0000-0002-5318-5526 